\title{
Information Security in Healthcare Organizations using Low-interaction Honeypot Intrusion Detection System
}

\author{
Aastha Yadav ${ }^{1}$, Sarthak Raisurana ${ }^{2}$, H. Balaji*, P. Lalitha*, \\ Ronnie D. Caytiles** and N. Ch. S. N. Iyengar* \\ ${ }^{1-2}$ VIT University, Vellore-632014, Tamil Nadu \\ **Department of Multimedia Engineering, Hannam University, Korea, \\ ${ }^{*}$ Sreenidhi Institute of Science and Technology Yamnampet. \\ Ghatkesar Hyderabad, India \\ \{aasthay1705, sarthak.dbz',rdcaytiles,nchsniyengar48\}@gmail.com
}

\begin{abstract}
Healthcare Organizations have seen an alarming rise in cyber-attacks in the recent years. One way a hacker could get control was by breaking into a medical network to gain access over the active medical devices that patients rely on for their survival. Our network model proposes a low-interaction and a medium-interaction honeypot based intrusion detection system using Dionaea and Kippo SSH to secure our internal network and study the activities of the intruders. We also look at a possible Metasploit attack and Brute force attack logged by Dionaea and Kippo SSH which prepares the Malware Analysis report of the suspicious file downloaded.
\end{abstract}

Keywords: low-interaction honeypot, medium-interaction honeypot, Doinaea, Kippo SSH, Metasploit attack, Brute force attack

\section{Introduction}

All organizational networks are vulnerable to a variety of exploits that can compromise their intended operations. The challenges of securing networks in the face of intruders have become overwhelming and are still growing. Honeypot Intrusion Detection System (IDS) is an attempt to overcome shortcomings of tradition systems towards a more safe and secure environment. A honeypot is a program, machine, or system put on a network as bait for attackers. The idea of the system is to deceive the attacker by making the honeypot seem like a legitimate system. Honeynets are useful to expose current vulnerabilities of the organization. Honeypots return highly valuable data that is much easier to interpret than that of IDS (Intrusion Detection System). The information gathered from honeypots is used by the administration to protect their system from potential attacks [2].

Dionaea is the successor of Nepenthes and is used as a malware capturing honeypot initially developed under The Honeynet Project's 2009 Google Summer of Code (GSoC). Dionaea's job is to trap malware exploiting vulnerabilities exposed by services offered over a network, and ultimately obtain a copy of the malware in the binaries and analyze them. Server Message Block (SMB) is the main protocol offered by Dionaea. SMB has been able to capture remote exploitable bugs, and is a very popular target for worms. The system supports HTTP and HTTPS on port 80. Dionaea provides a basic FTP server on port 21. It allows creation of directories, and uploading and downloading of files. Dionaea provides a TFTP server on port 60 which can be used to serve files. Dionaea implements the Tabular Data Stream protocol which is used by Microsoft SQL Server. Developed as part of GSoC 2011 by PhiBo, the VoIP protocol used in Dionaea is the Session Initial

Received (May 10, 2017), Review Result (September 15, 2017), Accepted (September 23, 2017) 
Protocol (SIP). This module does not connect to an external VoIP registrar / server, rather it simply waits for incoming SIP messages to log all data as incidents or binary data dumps, and reacts accordingly. Dionaea uses LibEmu to detect and evaluate payloads sent by attackers in order to obtain a copy of the malware. LibEmu is used detect, measure, and if necessary, execute the shellcode. Shellcode measurement / profiling are performed by executing the shellcode in LibEmu VM, and recording API calls and arguments [3].

\section{Motivation}

Ethical health research and privacy protections both provide valuable benefits to society. Health research is vital to improving human health and health care. Protecting patients sensitive data involved in research from the harms caused by breached information is essential to ethical research. The primary reason for protecting personal privacy is to protect the interests of individuals. Protecting the security of data in health research is important because healthcare organization requires the collection, storage, and use of large amounts of personally identifiable health information, much of which may be sensitive and potentially embarrassing. Healthcare data has been breached to sell for ransom as the recent trends of cyber-crimes have shown. Recent trends have also shown attacks being performed requires a complete analysis of hacker's activity to be tracked to secure the very sensitive healthcare information. This calls for building an Intrusion Detection System (IDS) such as honeypots that keeps track of hacker's moves and to move towards more secure network architecture.

\section{Background Research}

\subsection{Summary}

We propose using honeypots as a form of intrusion detection in the networks of healthcare organizations to keep patient data secure. Existing Intrusion Detection Systems:

\section{NETWORK BASED:}

Network-based Intrusion Detection Systems (NBIDS) are just what the name implies, "Network Based". This system uses a device that is directly connected to a network segment to monitor traffic flows. The device uses these traffic flows as it's data source to determine whether the traffic matches a known attack signature or pattern. The three main signatures that the NBIDS uses are; attack text string, port signatures, and header signatures. By using the network as a data source, the NBIDS give the ability to monitor entire segments of the network for malicious behavior.

\section{HOST BASED:}

Host-Based Intrusion Detection Systems (HBIDS) are another type of IDS to be considered. HBIDS typically consist of loading software on the system being monitored. The software monitors the system for changes resembling an attack or threat. HBIDS uses $\log$ files, auditing agents, communication traffic, system file integrity, suspicious processes, and user privileges to determine threats and attacks. Because the system is monitoring the individual host, it is effective in detecting isolated attacks including trusted-insider attacks [12].

\section{Different models of Intrusion detection:}

Intrusion Detection Systems also vary in way they determine an attacks and threat. The most prevalent models used to detect attacks include algorithms for statistical anomaly 
detection, rules-based detection, and a hybrid of the two. As with the type of IDS, the different models have advantages and disadvantages associated with each. The concept is to deploy the model that is most effective in the environment in which it will be used.

Statistical-anomaly model does just as the name implies, it looks for statistical abnormalities. This model runs under the assumption that abnormal behavior is indicative of a threat. The Statistical-anomaly model uses factors such as log files, audits, file/folder properties, and traffic patterns to determine normal system behavior. The key to the statistical-anomaly model is what the systems considered normal behavior. Also, we must determine how much suspicious behavior must deviate for the normal profile to be considered an attack. Deviation from normal activities is the basis for this IDS model.

Rule or Signature-based model: Most attacks are characterized by a sequence of events, making it is possible to create signatures to define these threats. The Signaturebased system examines its data source for matches to predefined signatures or activities. The system alarms attack matches to the signatures are found in the data. This model is easier to implement and maintain than the anomaly model. As the system has very specific events that it is searching for, it has a very low false positives rate in comparison to Anomaly based IDS [13].

\subsection{Gaps Identified}

Network based: Although the NBIDS is good for detecting broad network attacks or threats, it does have some drawbacks. Because the system is monitoring the network, it may not detect isolated attacks or threats. Therefore, NBIDS isn't as effective for detecting things such as trusted-insider attacks that may only target specific devices. So if one individual machine is compromised, it may not be detected if it isn't passing suspicious traffic over the network. Also, if an attack is disguised in legitimate network traffic such as HTTP, FTP, SMTP, etc., it could potentially be missed. So although the NBIDS does have drawbacks, it can be an effective security monitoring device to complement existing security measures.

Host Based: One drawback of the Host-based system is software must be installed and monitored on individual devices. In a large environment, this could become overwhelming [13].

Statistical Anomaly: The driving force in anomaly IDS is the use of abnormalities for detection. For this detection to occur, normal behavior must be identified. This normal behavior profile can either be manually created or can be adaptively learned. If the profile is created manually, it must be updated as the system evolves so it doesn't become outdate. Alternatively, if the profile is adaptively learned, there is an increased risk of false positives indicating an attack when one isn't present. Because the anomaly based system works off of a normal profile to detect abnormalities, it is a very customizable model for an organization to use. Along with the customization come high false-positive rates as well as high maintenance to update the "normal" profile.

Rule or Signature-based model: It can only detect attacks for which it has signatures. This need for signatures causes the system to be unable to detect new threats or "Zero Day" attacks [14].

\section{Healthcare Organizations: Vulnerable Sector of Cyber-attacks}

According to a research earlier this year with the Ponemon Institute, within healthcare and pharmaceutical companies, an average of $30 \%$ of outbound web traffic is encrypted 
today and these organizations expect that percentage to increase to $48 \%$ over the next 12 months. Indeed, healthcare organizations have been taking a multipronged approach, using a combination of people, policies and technical controls to combat cyber-attack and protect information, with encryption being considered as a best practice for protecting the electronic medical records (EMR) and personal health information (PHI) of patients [4]. A study by Experian found that Electronic health records remain likely to be a top target for hackers [10]. Despite the fact that healthcare organization will more likely be taking far harsher cyber security precautions which will ward off lethal attacks, the growing proliferation of cheap, connected Internet of Things (IoT) devices will provide an easy gateway for criminals to illegally access critical information and personal data [4].

In a recent SANS survey, the findings of this study indicate that 7 percent of traffic was coming from radiology imaging software, another 7 percent of malicious traffic originated from video conferencing systems, and another 3 percent came from digital video systems that are most likely used for consults and remote procedures. In this study, most of the malicious traffic passed through or was transmitted from VPN applications and devices ( 33 percent), 13 whereas 16 percent was sent by firewalls, 7 percent was sent from routers and 3 percent was sent from enterprise network controllers (ENCs). This indicates that the security devices and applications themselves were either compromised, which is a common tactic among malware families, or that these "protection" systems are not detecting malicious traffic coming from the network endpoints inside the protected perimeter-inside the firewall or behind the VPN concentrator. If they are not detecting, they are not reporting - and that means they are out of compliance with privacy and security regulations for patient data. This report, however, shows that the systems were compromised for long periods of time, and even when alerted to their system's actions, the organizations did not repair the vulnerabilities [5].

\section{Proposed Architecture of Network Design}

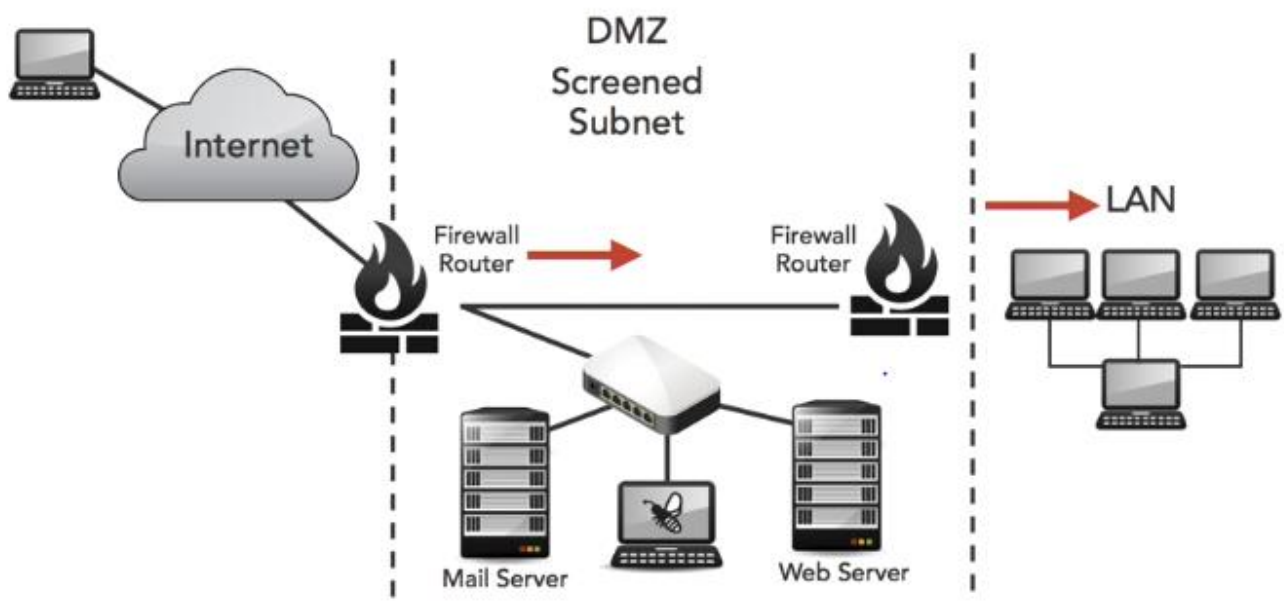

Figure 1. Architecture of Proposed Network Design

The above proposed model will make use of low-interaction and a mediuminteraction Virtual Honeypot to collect malware in an automated way. A lowinteraction honeypot provides only limited access to the operating system. By design, it is not meant to represent a fully featured operating system and usually cannot be completely exploited. As a result, a low-interaction honeypot is not well suited for capturing zero-day exploits. Instead, it can be used to detect known exploits and measure how often your network gets attacked. The advantages of low-interaction honeypots are manifold. They 
are easy to set up and maintain. They do not require significant computing resources, and they cannot be compromised by adversaries. The risk of running low-interaction honeypots is much smaller than running honeypots that adversaries can break into and control. On the other hand, that is also one of the main disadvantages of the lowinteraction honeypots. They only present the illusion of a machine, which may be pretty sophisticated, but it still does not provide an attacker with a real root shell [6].

The aim of this project is to look at an approach to collect malware with the help of honeypots and to monitor activities of the intruder on the shell of a fake system. This will help us build an Intrusion Detection System (IDS) against malware which in the form of botnets can bring down almost any server through Distributed Denial of Service (DDoS), the combined power of many compromised machines is a constant danger even to uninfected sites.

Honeypots in the DMZ, which are exposed to external traffic, will detect external attacks and probes. Given the current amount of noise in the Internet this will probably amount for lots of unimportant probes and scans together with the important ones. On the other hand, honeypots in the internal network would detect internal attacks, either true malicious activity or just bad traffic generated by infected or misconfigured systems [11]. As most of the attacks in a healthcare organization are performed by insiders, it would be ideal to place the honeypot inside LAN to catch any malware or malicious activity.

\section{Honeypot: An approach to Collect Malware}

The proposed system uses Dionaea and Kippo SSH as the honeypot system to collect malware. Dionaea's intention is to trap malware exploiting vulnerabilities exposed by services offered to a network and to achieve the goal of downloading malware. Dionaea uses libev to get notified once it can act on a socket, or begin to read or write. It can offer services via tcp/udp and tls for IPv4 and IPv6, and can apply rate limiting and accounting limits per connections to tcp and tls connections - if required. Dionaea works on a number of modules. Honeypot listens on all available interfaces in the network on the organization. The curl module is used to transfer files from and to servers, it is used to download files via http as well as submitting files to 3rd parties. The emu module is used to detect, profile and to execute shellcode.

Nowadays worms use API to access services, before sending their payload. To allow easy adjustments to the protocol, dionaea implements the protocols in python. There is glue between the network layer which is done in the c programming language and the embedded python scripting language, which allows using the non-blocking connections in python.

Attackers attempt is not to seek your service, but exploit you; they'll chat with the service for some packets, and afterwards send a payload. Dionaea has to detect and evaluate the payload to be able to gain a copy of the malware. In order to do so, dionaea uses libemu. The part of dionaea which takes care of the network io can create a copy of all in/output run for a connection, this copy is passed to the detection facility, which is a tree of detection facilities, at this moment there is only a single leaf, the emu plugin. The emu plugin uses threads and libemu to detect and profile/measure shell code [7]. Dionaea is considered to be the successor of Nepenthes and the aforementioned visualization tool utilizes its XMPP backend in order to present some statistics of the honeypot's activity. The creator of Dionaea has included some rather basic visualization tools in the software, but these proved to be infective when the dataset grows large in size [8].

Kippo is a medium interaction SSH honeypot designed to log brute force attacks and, most importantly, the entire shell interaction performed by the attacker. SSH protocol can be used to perform a secure remote login over an insecure network to access a remote shell. In order to study the activities performed by attackers after they compromised a system with an SSH server, we can use a Kippo honeypot. Kippo allows an attacking 
entity to attempt a login to the system, believing it is entering into a legitimate SSH session with the server. Now the attacker tries to guess the password and upon successful guessing of the password, the attacker is then moved into a fake system with which they can interact. Kippo SSH honeypot is placed before any administrative systems so the attacker is logged on to it assuming it's a legitimate system. In this fake system, all interactions with the shell are monitored and recorded. The system also allows the use of wget and other commands commonly used to fetch or download files. The main objective of the implementation is to bring to the attacker the impression of navigating the real system of the organization. IT security can give a view on the basis of the command given by him - for what purpose it was hacked, which data the attacker was trying to get, and what techniques were used [15].

\section{Healthcare Cyber Security Attacks}

Some 93 major cyber-attacks hit healthcare organizations this year, up from 57 in 2015, new research shows. Sophisticated attackers are now responsible for $31 \%$ of all major HIPAA data breaches reported this year, a 300\% increase over the past three years, according to the report. Cybercriminals were responsible for $10 \%$ of all major data breaches in 2014 and $21 \%$ in 2015 [1]. Here's a list of sample medical equipments which when hacked by an intruder can cause serious situations.

\subsection{X-ray Generator}

Medical X-ray machines are used to take pictures of dense tissues. The radiation from X-ray machines are highly penetrating, ionizing radiation, therefore they can be very dangerous. If a hacker is able to increase the dose or radiation exposure, a patient could be overexposed to enough radiation that it results in permanent destruction of either hair or sweat glands, or skin with a resulting scar. The irreversible changes are categorized as radiation dermatitis, chronic radiation dermatitis and radiation cancer. Acute exposure is a one-time event with high-level dose (>100 rem or 1 Sievert) and symptoms appear quickly (within days or weeks). Also, Long-term effects of chronic exposure to ionizing radiation include carcinogenesis, life span shortening, and cataract formation with the principle delayed effect being an increased incidence of leukemia and other cancers.

\subsection{Dialysis Machine}

A dialysis machine is designed to replace many of the kidney's important functions and restore a patient's blood to a normal, healthy balance by filtering out harmful wastes, salt, and excess fluid. A doctor creates a vascular access into the patient's blood vessels so the patient can be connected to the filtering machine during each hemodialysis session. In a dialysis machine, two independent failures are required before the machine operates in an unsafe condition. A single failure is probably not life threatening. Two independent failures, however, would be life threatening-not a coincidence. If the hacker spoofs extracorporeal venous pressure decrease, biomedical technician assumes disconnect of needle from patient vascular access.

There are many more such attacks that can be performed on medical equipment such as infusion pump, barcode scanning systems, Medical imaging systems by simple spoofs and altering or modifying the frequencies or data causing fatal damage to the health of a patient [9]. 


\section{Implementation}

We run our simulations to capture malware on our honeypot system. We present statistics about the collected binaries by analyzing the activity and behavior of malware using dockers to study the types of malware. VmWare workstation is used to create the virtual system and Ubuntu 14.04 is installed on it. The computer and operating system instance that executes the VMware process is referred to as the host machine. In order to run Linux-based honeypots, we are running Ubuntu 64-bit as the guest operating system. For each virtual machine, we allocated budget at least 256MB RAM or, even better, 512MB.

Dionaea gains location of the file the attacker wants it to download from the shell code and it tries the download the file. The protocol to download files via tftp and ftp are implemented in python (ftp.py and tftp.py) as a part of Dionaea. Dionaea can then http/POST the file to several services like CWSandbox, Norman Sandbox or VirusTotal.

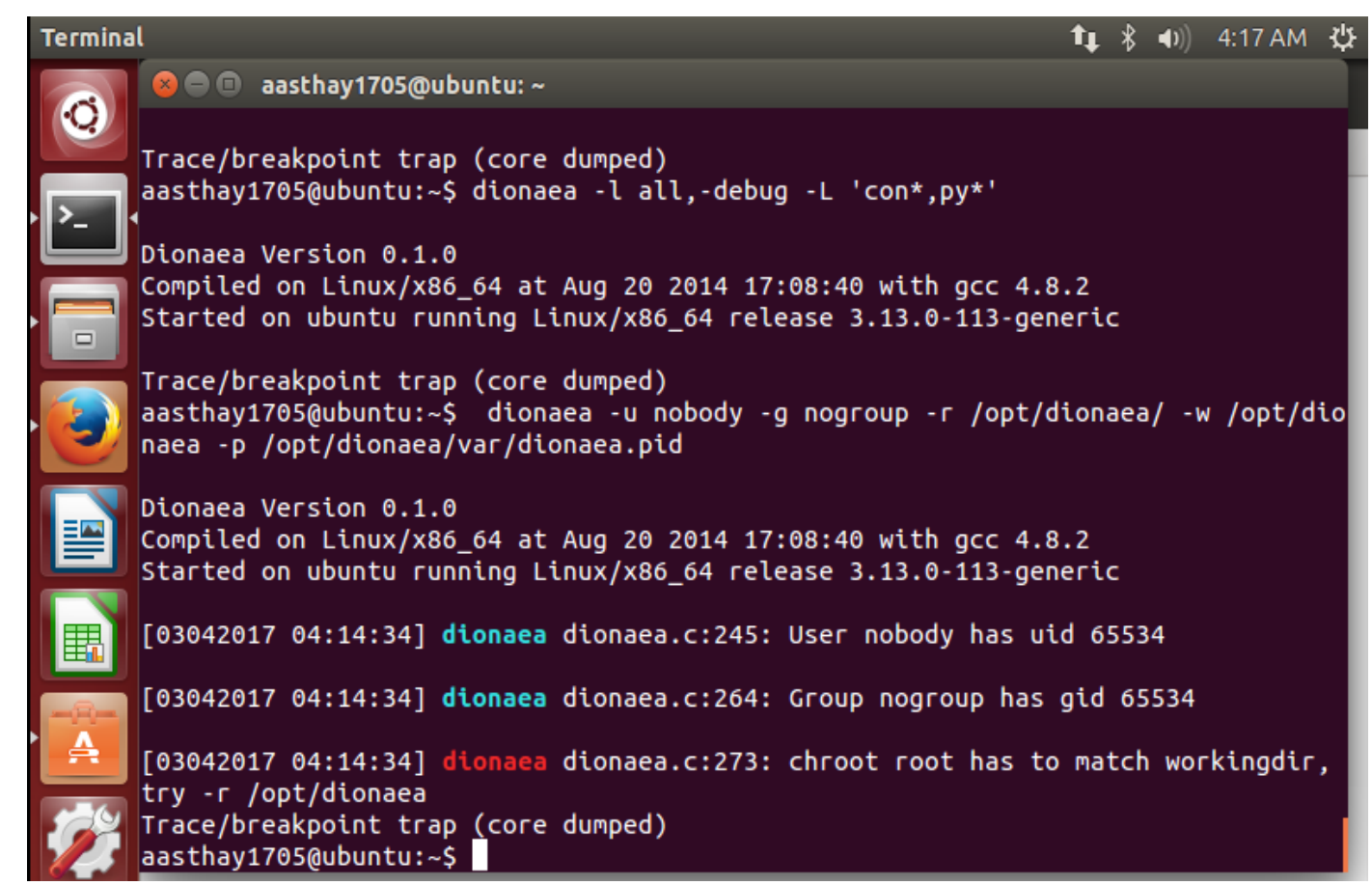

\section{Figure 4. Dionaea Setup to Create User and Group}

We used our Dionaea setup to perform a metasploit attack and check the dionaea.log to see it logs the information. Attackers do not seek your service, attackers want to exploit you, they'll chat with the service for some packets, and afterwards sent a payload. dionaea has to detect and evaluate the payload to be able to gain a copy of the malware. In order to do so, dionaea uses libemu. 


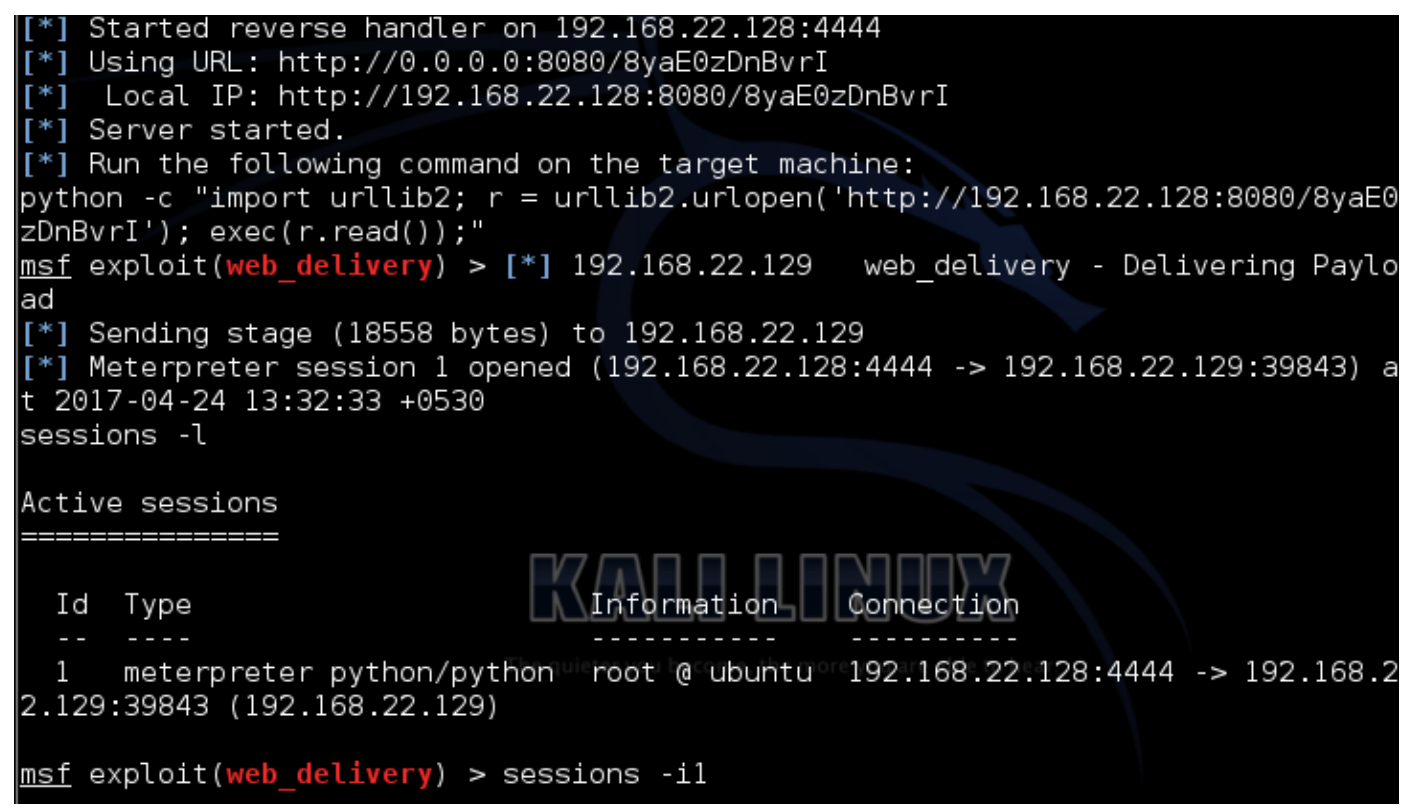

Figure 5. Metasploit Exploitation Performed

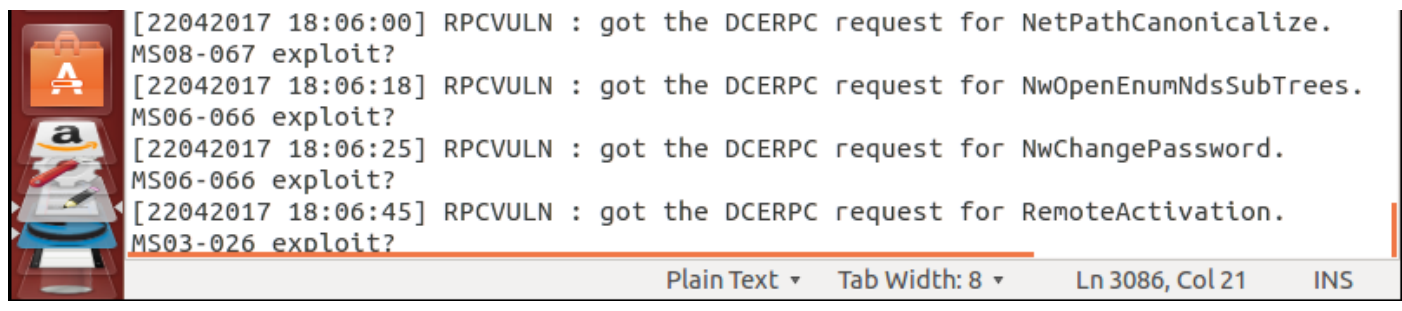

Figure 6. Dionaea.log Spits Out the Information

The honeypot Dionaea also already supports shell emulation and ftp/http/tftp downloads of malware. From figure 5, the script command generated by this exploit on the target, we are able to get complete control of the system including keystroke logging, turning on microphone to hear and reading or deleting any files on the system. SMB is the main protocol offered by Dionaea. SMB has a decent history of remote exploitable bugs, and is a very popular target for worms. And, Figure 6 is the log file of Dionaea that logs a possible MS08-067 exploit.

Dionaea also has a virustotal module to automatically submit the suspicious files and prepare a malware analysis report for the same. In the figure below (7), the file downloaded from the url in Figure 5, undergoes virustotal scan for malware behaviour. 


\section{¿viliustotal}

\begin{tabular}{|c|c|c|c|}
\hline SHA256: & \multicolumn{2}{|c|}{ 127d1cd82f4c6697eb371dcf5619481606cdef43139b07692f4beb2b59b3ea8c } & \\
\hline File name: & \multicolumn{2}{|l|}{ Qarawlfy } & \\
\hline Detection ratio: & \multicolumn{2}{|l|}{$3 / 55$} & \\
\hline Analysis date: & \multicolumn{2}{|c|}{ 2017-04-24 11:43:17 UTC ( 0 minutes ago ) } & \\
\hline 国 Analysis & (3) Additional information & Comments PVotes & \\
\hline Antivirus & & Result & Update \\
\hline Avast & & JS:Downloader-EQA [Tri] & 20170424 \\
\hline ClamAV & & Legacy.Trojan.Agent-37025 & 20170424 \\
\hline Qihoo-360 & & Script/Trojan.Downloader.4e1 & 20170424 \\
\hline
\end{tabular}

Figure 7. VirusTotal Scan of the File

Next we track malicious activity of an intruder with Kippo SSH set up on port 22.

From the figure 8, we can see the intruder's activity logged on kippo.log. This includes username and passwords entered including the add, modify and deleting commands run by the intruder on the files of the fake file system. The system also allows the use of wget and other commands commonly used to fetch or download files, as well as a base set of utilities. It saves the files downloaded with wget for later investigation $\mathrm{dl}$ folder of logs.

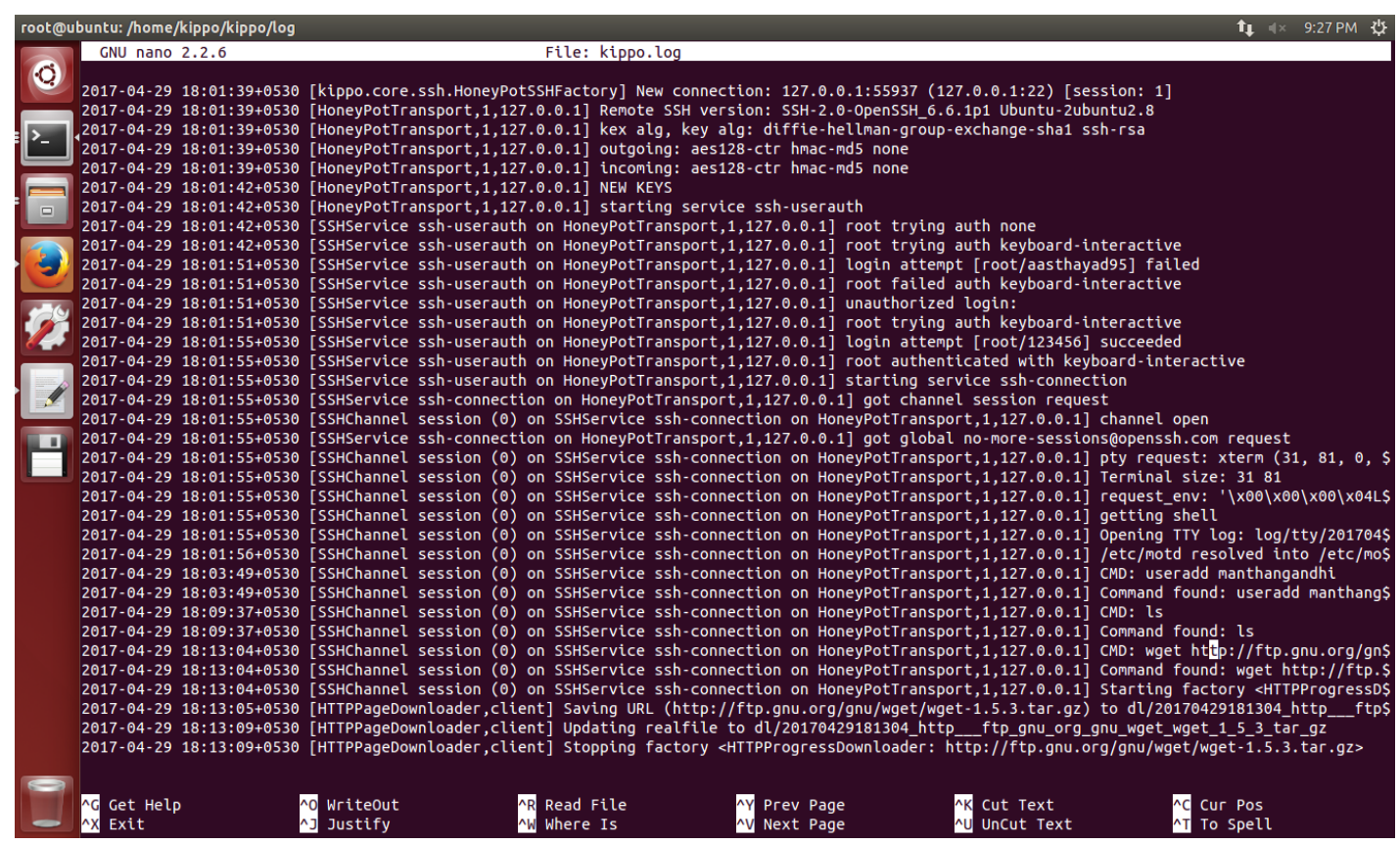

Figure 8. Intruder's Activity on Kippo on the Fake File System 
Table 1. Kippo's Logged Contents

\begin{tabular}{|c|c|}
\hline $\begin{array}{c}\text { Essential } \\
\text { Information }\end{array}$ & Log File \\
\hline wget commands & $\mathrm{d}$ l/ \\
\hline Username attempt & mysql.sql \\
\hline Password attempt & mysql.sql \\
\hline Session ID & log/tty/ \\
\hline Session Timestamp & log/tty/ \\
\hline $\begin{array}{c}\text { Fake file system } \\
\text { contents }\end{array}$ & honeyfs/ \\
\hline
\end{tabular}

Table 1 includes the location of all the essential information logged about the intruder's activity on the fake file system .

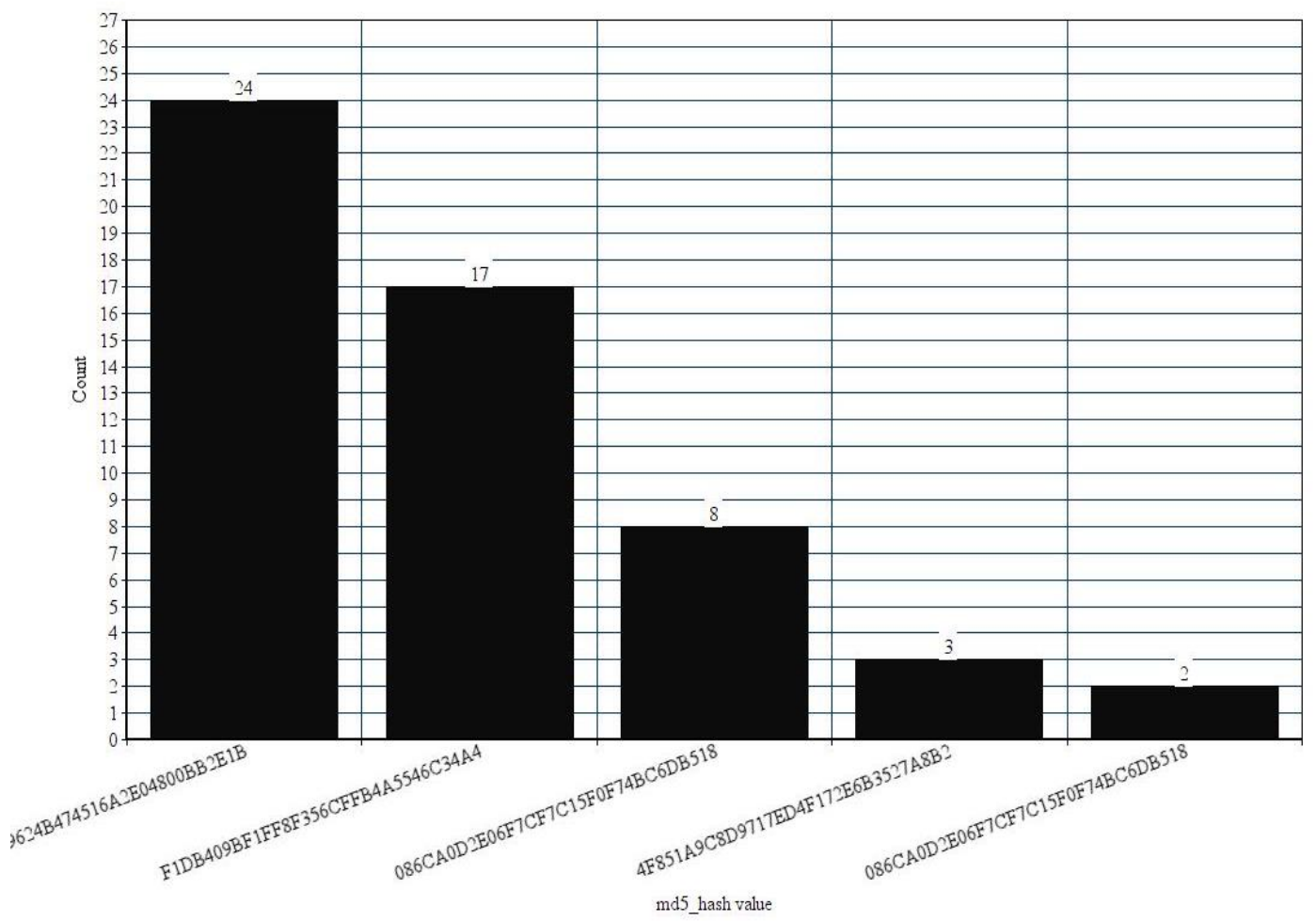

Figure 9. md5_hash Value of the Malware Downloaded

From the above Figure (9), we can get the md5_hash value downloaded over a period of 2 months by the honeypot Dionaea up and running in the binaries folder. Dionaea successfully logs all the payloads and shell binds and downloads the malware for further analysis. 
Table 2. Honeypot Systems as Security Mechanism

\begin{tabular}{|c|c|c|c|}
\hline $\begin{array}{l}\text { Honeypot } \\
\text { System }\end{array}$ & $\begin{array}{l}\text { Hacker } \\
\text { Activity }\end{array}$ & $\begin{array}{l}\text { Potential } \\
\text { Vulnerability/Hackable } \\
\text { Device }\end{array}$ & Honeypot Solution \\
\hline Dionaea & $\begin{array}{l}\text { Send an email } \\
\text { with a payload } \\
\text { using } \\
\text { Metasploit } \\
\text { Exploit that } \\
\text { runs on the } \\
\text { medical device }\end{array}$ & $\begin{array}{l}\text { Exposed Networking gear } \\
\text { and admin computers of } \\
\text { Healthcare organizations } \\
\text { to exploit critical medical } \\
\text { devices }\end{array}$ & $\begin{array}{l}\text { Logs the downloaded } \\
\text { payloads and } \\
\text { performs the analysis } \\
\text { on malware detected } \\
\text { using VirusTotal, } \\
\text { CWSandbox etc. }\end{array}$ \\
\hline $\begin{array}{l}\text { Kippo } \\
\text { SSH }\end{array}$ & $\begin{array}{l}\text { Successful SSH } \\
\text { and web logins } \\
\text { on critical } \\
\text { medical devices }\end{array}$ & $\begin{array}{l}\text { Secure Server of the } \\
\text { organization }\end{array}$ & $\begin{array}{l}\text { Logs the activity of } \\
\text { the intruder including } \\
\text { IP activity, geological } \\
\text { location, inputs, } \\
\text { password and } \\
\text { usernames tried on } \\
\text { the fake file system. }\end{array}$ \\
\hline
\end{tabular}

Table 2 explains how honeypots act as a security mechanism to intruder's activity in an attempt to gain access to medical devices to modify or sweep away patient's critical information.

\section{Conclusion}

The work concludes that Dionaea and Kippo SSH honeypot system act as an effective security mechanism placed in the DMZ to trap malware and to perform reports of malware ananlysis on logged binaries. They can also be used to log all activities of an attacker on the shell. This system can also be used to send emails to the administrative department in the healthcare organization so that they may be notified of the intruder's activities. Cybersecurity knowledge for healthcare orgainizations is important for the hospital staff. They have to be trained to understand the aspects of it. A secure and safe environment for patients is the goal of cybersecurity.

\section{References}

[1] "Major Cyberattacks On Healthcare Grew 63\% In 2016", http://www.darkreading.com/attacksbreaches/major-cyberattacks-on-healthcare-grew-63--in-2016/d/d-id/1327779

[2] B. Hancock and J. W. Rittinghouse, "Cybersecurity Operations Handbook: The Definitive Reference on Operational Cybersecurity", Elsevier Science Inc., (2003).

[3] Dionaea, "A Malware Capturing Honeypot", https://www.edgis-security.org/honeypot/dionaea/

[4] "Why healthcare is a vulnerable sector for cyber attack - and what can be done about it". Available: http://www.appstechnews.com/news/2017/jan/17/why-healthcare-vulnerable-sector-cyber-attack-andwhat-can-be-done-about-it/

[5] "Health Care Cyberthreat Report: Widespread Compromises Detected, Compliance Nightmare on Horizon", Available: https://www.sans.org/reading-room/whitepapers/analyst/health-care-cyberthreatreport-widespread-compromises-detected-compliance-nightmare-horizon-34735

[6] N. Provos , T. Holz, "Virtual honeypots: from botnet tracking to intrusion detection ", Addison-Wesley Professional, (2007).

[7] Dionaea, "Documentation", Available:http://dionaea.readthedocs.io/en/latest/old/exploitation.html

[8] I. Koniaris, G. Papadimitriou, P. Nicopolitidis, M. Obaidat, "Honeypots Deployment for the Analysis and Visualization of Malware Activity and Malicious Connections", (2014).

[9] L.Ayala, "Cybersecurity for Hospitals and Healthcare Facilities: A Guide to Detection Facilities", pp 2129.

[10] "Healthcare top target for cyberattacks in 2017, Experian predicts", Available: http://www.healthcareitnews.com/news/healthcare-top-target-cyberattacks-2017-experian-predicts

[11] David Pérez Conde, "Deploying Honeypots and the Security Architecture of a Fictitious Company", February 2005, pp 11 
[12] Lata, Indu Kashyap, "Study and Analysis of Network based Intrusion Detection System", vol. 2, no. 5, (2013).

[13] "Intrusion Detection Systems in Hospitals - Infosec Writers", http://www.infosecwriters.com/text_resources/pdf/IDS_JBarnes.pdf

[14] "Anomaly Based Intrusion Detection System: Wikipedia", https://en.wikipedia.org/wiki/Anomalybased_intrusion_detection_system

[15] "Tracking Attackers with a Honeypot - part 2", (Kippo), http://resources.infosecinstitute.com/trackingattackers-honeypot-part-2-kippo/\#gref

\section{Authors}

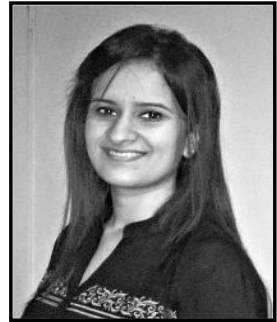

Aastha Yadav, is a student, currently pursuing Masters in Cybersecurity at Syracuse University, Syracuse NY. Her research interests include Network Security and Knowledge based Artificial Intelligence. She is a computer enthusiast and has keen interest in developing secure software solutions for problems. She loves working on projects with real life application and scope using computer applications.

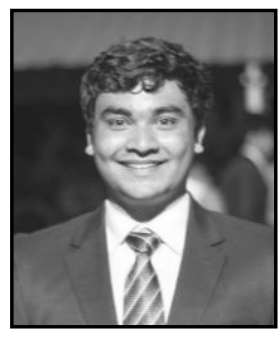

Sarthak Raisurana is a Business Technology Analyst at ZS Associates, Pune, Maharashtra, India. His interests lie in the fields of Mathematical Cybersecurity (cryptography) and Machine Learning. He likes thinking of abstract and Hypothetical problems, and coming up with solutions to them.

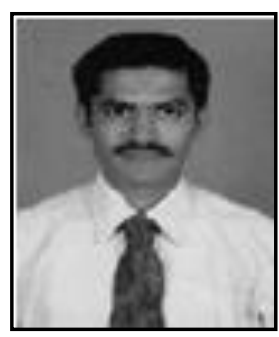

H. Bajaj currently working as an Associate Professor in Computer Science and Engineering Department at Sreenidhi Institute of Science and Technology, Hyderabad, Telangana India. His areas of research include Data Warehousing and Mining and Network Security.

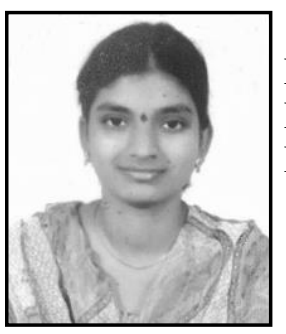

P. Lalitha currently working as an Asst.Professor in IT Department at Sreenidhi Institute of Science and Technology, Hyderabad, Telangana India. Her areas of research include Information Security and DBMS. 


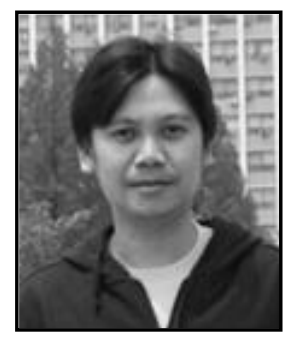

Ronnie D. Caytiles, he had his Bachelor of Science in Computer Engineering- Western Institute of Technology, Iloilo City, Philippines, and Master of Science in Computer Science- Central Philippine University, Iloilo City, Philippines. He finished his Ph.D. in Multimedia Engineering, Hannam University, Daejeon, Korea. Currently, he serves as an Assistant Professor at Multimedia Engineering department, Hannam University, Daejeon, Korea. His research interests include Mobile Computing, Multimedia Communication, Information Technology Security, Ubiquitous Computing, Control and Automation

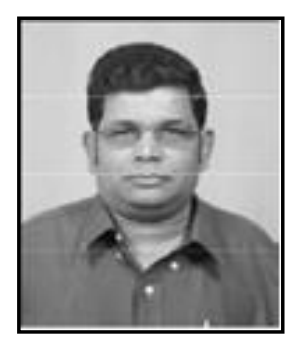

N. Ch. S. N. Iyengar (b 1961), he currently Professor at the Sreenidhi Institute of Science and Technology (SNIST) Yamnapet, Ghatkesr, Hyderabad, Telengana, India. His research interests include Agent-Based Distributed Computing, Intelligent Computing, Network Security, Secured Cloud Computing and Fluid Mechanics. He had 32+ years of experience in teaching and research, guided many scholars, has authored several textbooks and had nearly 200+ research publications in reputed peer reviewed international journals.He served as PCM/reviewer/keynote speaker/ Invited speaker. 
International Journal of Security and Its Applications

Vol. 11, No. 9 (2017) 